\title{
Correction to: Silk Fibroin Bioinks for Digital Light Processing (DLP) 3D Bioprinting
}

Soon Hee Kim, Do Yeon Kim, Tae Hyeon Lim, and Chan Hum Park

\section{Correction to:}

Chapter 4 in: H. J. Chun et al. (eds.), Bioinspired Biomaterials, Advances in Experimental Medicine and Biology 1249, https://doi.org/10.1007/978-981-15-3258-0_4

An earlier version of the chapter was published with an incorrect Acknowledgements text which was corrected as follows:

This work was supported by the National Research Foundation of Korea (NRF) grant funded by the Korea government (MSIP) (grant No.: 2016R1E1A1A01942120), Republic of Korea and by the Hallym University Research Fund. 